\title{
Polytraumaversorgung als Bereich der hochspezialisierten Medizin
}

\author{
Hans-Peter Simmena,e, Kai-Oliver Jensen ${ }^{b, e}$, Kai Sprengel ${ }^{c, e}$, Hans-Christoph Pape ${ }^{d, e}$

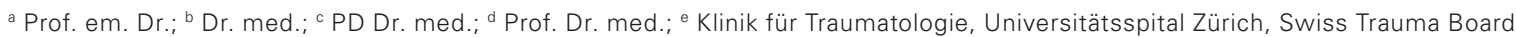

Schwerverletzte Patientinnen und Patienten werden in der Schweiz im Rahmen der interkantonalen Vereinbarung der hochspezialisierten Medizin (IVHSM) behandelt. Das Swiss Trauma Board und die dort vereinten zwölf Schweizer Traumazentren arbeiten unter anderem mit dem deutschen Traumaregister zusammen, um eine bestmögliche Behandlung der Verletzten zu gewährleisten.

Im Jahr 2008 unterzeichneten alle Schweizer Kantone die interkantonale Vereinbarung der hochspezialisierten Medizin (IVHSM). Die dort vermerkten Pflichtregister erlauben die Überprüfung der HSM(hochspezialisierte Medizin)-Leistungen in den Spitälern mit Leistungsauftrag. Im Rahmen der interkantonalen Planung der hochspezialisierten Medizin wurde die Behandlung schwerverletzter Erwachsener 2011 erstmals als medizinischer Bereich an zwölf ausgewählte HSM-Traumazentren übertragen [1-3].

\section{Zusammenarbeit mit deutschem Traumaregister}

Die gesundheitliche Prognose von schwerverletzten Personen hängt entscheidend von einer zeitnahen, adäquaten und prioritätengerechten Diagnose und Therapie ab. Bei Verdacht auf eine schwere Verletzung sollen aus diesem Grund der Patient oder die Patientin einem HSM-Traumazentrum zugewiesen werden.
Über ein solch spezialisiertes Traumazentrum verfügen die fünf Universitätsspitäler sowie die Kantonsspitäler Aarau, Chur, Lugano, Luzern, St. Gallen, Sitten und Winterthur. Diese Verteilung berücksichtigt die geographischen und sprachlichen Verhältnisse der Schweiz in angemessener Weise. Um ihrem Auftrag nachkommen zu können, müssen die zwölf Zentren über eine vorgeschriebene beträchtliche Infrastruktur und über ebenfalls definierte personelle Ressourcen verfügen, was sehr kostspielig ist [4].

Das «Swiss Trauma Board», die Vereinigung der zwölf Traumazentren, ist seit 2011 als Gremium organisiert. Der Vorsitzende während der ersten vier Jahre war Marcel Jakob aus Basel, seit 2015 amtet Hans-Peter Simmen aus Zürich in dieser Position [5]. Das Gremium trifft sich mehrmals jährlich und arbeitet freundschaftlich und kollegial zusammen, um die Behandlung von Schwerverletzten weiterzuentwickeln. Eine vom HSM-Beschlussorgan dem «Swiss Trauma Board» übertragene Hauptaufgabe war die Entwicklung eines

\section{Das Wichtigste in Kürze}

- Schwerverletzte Patientinnen und Patienten werden in der Schweiz im Rahmen der interkantonalen Vereinbarung der hochspezialisierten Medizin (IVHSM) behandelt.

- Das sogenannte "Swiss Trauma Registry" (STR) kooperiert mit dem deutschen Traumaregister (TraumaRegister DGU ${ }^{\circledR}$ ), um nicht nur eine interne, sondern auch eine externe Evaluation zu gewährleisten.

- Die offene und detaillierte gemeinsame Analyse der jährlichen Berichte - eine zentrale Aufgabe des "Swiss Trauma Board" - zeigt, dass die Rettungssysteme in der Schweiz, insbesondere die Luftrettung, und die Behandlungsqualität im Vergleich zu ausländischen Registern sehr gut sind.

\section{L'essentiel en bref}

- Les patients gravement blessés sont traités en Suisse dans le cadre de la Convention intercantonale relative à la médecine hautement spécialisée (CIMHS).

- Le "Swiss Trauma Registry» (STR) coopère avec le Registre allemand des traumatismes (TraumaRegister $\mathrm{DGU}^{\circledR}$ ) pour assurer une évaluation à la fois interne et externe.

- L'analyse commune des rapports annuels effectuée ouvertement et en détail - une tâche centrale du "Swiss Trauma Board" - montre que les systèmes de secours en Suisse, en particulier le secours aérien, et la qualité du traitement sont très bons en comparaison avec les registres étrangers. 


\begin{tabular}{|c|c|c|}
\hline & $\begin{array}{c}\text { "Dein Spital" } \\
2017-2019\end{array}$ & $\begin{array}{c}\text { G-STC } \\
2017-2019\end{array}$ \\
\hline $\begin{array}{c}\text { Patient/innen } \\
\text { in der } \\
\text { Benchmark- } \\
\text { Gruppe }\end{array}$ & $\begin{array}{c}100 \% \\
(N=2,777)\end{array}$ & $\begin{array}{c}100 \% \\
(N=17,834)\end{array}$ \\
\hline Kopf & $\begin{array}{c}49.6 \% \\
(n=1,377)\end{array}$ & $\begin{array}{c}50.0 \% \\
(n=8,914)\end{array}$ \\
\hline Gesicht & $\begin{array}{c}18.7 \% \\
(n=519)\end{array}$ & $\begin{array}{c}18.6 \% \\
(n=3,323)\end{array}$ \\
\hline Hals & $\begin{array}{c}3.1 \% \\
(n=86)\end{array}$ & $\begin{array}{c}2.6 \% \\
(n=471)\end{array}$ \\
\hline Brustkorb & $\begin{array}{c}74.1 \% \\
(n=2,057)\end{array}$ & $\begin{array}{c}77.7 \% \\
(n=13,860)\end{array}$ \\
\hline Unterleib & $\begin{array}{c}31.4 \% \\
(n=872)\end{array}$ & $\begin{array}{c}28.1 \% \\
(n=5,004)\end{array}$ \\
\hline Wirbelsäule & $\begin{array}{c}44.6 \% \\
(n=1,239)\end{array}$ & $\begin{array}{c}45.2 \% \\
(n=8,067)\end{array}$ \\
\hline Arme & $\begin{array}{c}42.1 \% \\
(n=1,170)\end{array}$ & $\begin{array}{c}42.0 \% \\
(n=7,494)\end{array}$ \\
\hline Becken & $\begin{array}{c}30.0 \% \\
(n=833)\end{array}$ & $\begin{array}{c}39.9 \% \\
(n=5,338)\end{array}$ \\
\hline Beine & $\begin{array}{c}29.6 \% \\
(n=823)\end{array}$ & $\begin{array}{c}34.0 \% \\
(n=6,069)\end{array}$ \\
\hline
\end{tabular}

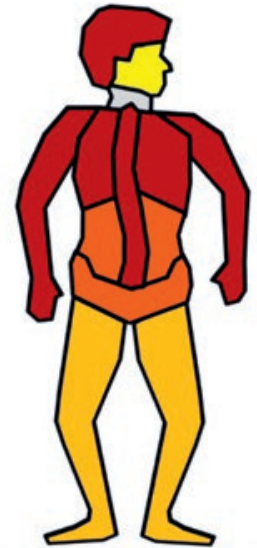

Verteilungsmuster der Verletzungen im STR für die Benchmark-Gruppe von 2017-2019
Mit «Dein Spital» ist die Gesamtheit des STR gemeint, "G-STC» meint das deutsche Traumaregister. Die Muster im Schweizer Register sind fast deckungsgleich mit dem deutschen Register. letzungsschwere in Punkten misst. Für den Einschluss in die HSM-Kriterien sind in der Schweiz 20 Punkte erforderlich. Pro Jahr erreichen etwa 2000 Personen 16 oder mehr Punkte, gegen 1800 Personen erreichen 20 Punkte und werden somit in die HSM-Beurteilung eingeschlossen. Bei den dort registrierten Unfallmechanismen handelt es sich fast ausschliesslich um stumpfe Krafteinwirkungen; Stich- und Schussverletzungen dagegen machen weniger als 3\% aus. Das durchschnittliche Alter der verletzten Personen liegt bei 58 Jahren, wobei 75\% davon Männer sind. Die Tendenz, dass das Alter der polytraumatisierten Personen steigt, ist schon seit einigen Jahren zu beobachten und entspricht der demographischen Entwicklung.

Es darf festgehalten werden, dass die Schweiz über ein sehr effizientes Rettungswesen verfügt; die Zeitspanne vom Unfall bis zum Eintreffen im Spital beträgt rund eine Stunde. Durchschnittlich werden 36\% der Patientinnen und Patienten mit dem Hubschrauber ins Spital gebracht, wobei natürlich landschaftsbedingt Unterschiede zu beobachten sind. Dem Kantonsspital Graubünden in Chur werden beispielsweise 60\% der Patien-

Traumaregisters zur Qualitätssicherung und Forschung. Nach ausgiebiger Diskussion im Jahre 2012 wurde beschlossen, dass das sogenannte «Swiss Trauma Registry" (STR) mit dem deutschen Traumaregister kooperieren soll (TraumaRegister $\left.\mathrm{DGU}^{\circledR}\right)$, um nicht nur eine interne, sondern auch eine externe Evaluation zu gewährleisten. Dieses Register existiert bereits seit über 25 Jahren und sammelt Daten von etwa 600 Kliniken. Eines der erklärten Hauptziele des STR ist der internationale Benchmark, der durch die Kooperation mit dem TraumaRegister $\mathrm{DGU}^{\circledR}$ nun möglich ist. Durch unterschiedliche Gewichtung einzelner Diagnosen, beispielsweise des isolierten Schädel-Hirn-Traumas, wird diese Zusammenarbeit derzeit noch erschwert - diese Probleme lassen sich jedoch lö-

Das Ziel des «Swiss Trauma Board»:

Schwerverletzten zeitnah die bestmögliche Therapie anzubieten.

sen. Es zeigt sich, dass die Verteilung der im «Swiss Trauma Registry" verzeichneten verletzten Körperregionen und damit das Vergleichskollektiv auf wenige Prozente mit dem riesigen Datensatz aus Deutschland übereinstimmt.

\section{Schweizer Rettungswesen ist effizient}

Eine Person gilt als schwerverletzt, wenn sie auf der ISS-Skala, dem «Injury Severity Score», 16 oder mehr Punkte erreichet. Der ISS ist ein System, das die Ver-
Die verletzten Körperregionen aus dem Schweizer Register stimmen fast genau mit dem riesigen Datensatz aus Deutschland überein.

tinnen und Patienten per Hubschrauber zugewiesen. Der genaue Unfallzeitpunkt lässt sich aber nicht immer feststellen und muss teilweise geschätzt werden. Verkehrsunfälle machen ungefähr 25\% der Vorkommnisse aus. Der durchschnittliche Spitalaufenthalt dauert 15 Tage, davon 6 Tage auf der Intensivstation. Die Mortalität der Patientinnen und Patienten im «Swiss Trauma Registry» beträgt etwa $12 \%$, was als indirektes Zeichen für ein qualitativ gutes Rettungswesen und gute Spitalbehandlung aufgefasst werden kann.

\section{Möglichst identische Quantifizierung der Daten angestrebt}

Das «Swiss Trauma Board» erarbeitete basierend auf international anerkannten Guidelines für Rettungsdienste Empfehlungen, in welcher Situation Patientinnen und Patienten direkt einem der zwölf Traumazentren zugewiesen werden sollten. Diese Empfehlungen wurden an das schweizerische Gesundheitssystem und an die hiesige Geographie angepasst. Dennoch wurde die Publikation dieser Empfehlungen zunächst nicht überall gut aufgenommen, weil mehrere grössere Spitäler befürchteten, man wolle ihnen Patienten wegnehmen. Schliesslich gelang es den Beteiligten darzulegen, dass das Ziel des "Swiss Trauma Board» 
sei, Schwerverletzten oder potenziell Schwerverletzten zeitnah die bestmögliche Therapie anzubieten, und dass regionale Interessen weniger zu gewichten seien. Inzwischen hat sich diese Problematik aus Sicht des «Swiss Trauma Board» gelegt $[6,7]$.

Die offene und detaillierte gemeinsame Analyse der jährlichen Berichte - eine zentrale Aufgabe des «Swiss Trauma Board» - zeigt, dass die Rettungssysteme in der Schweiz, insbesondere die Luftrettung, und die Behandlungsqualität im Vergleich zu ausländischen

Die Rettungssysteme in der Schweiz, besonders die Luftrettung, und die Behandlungsqualität sind im Vergleich zum Ausland sehr gut.

Registern sehr gut sind. Es gibt jedoch Unterschiede innerhalb der zwölf Schweizer Traumazentren, die nach Erachten der Autoren nicht auf qualitativen Differenzen beruhen, sondern auf die subjektive Erhebung der Daten zurückzuführen sind. Jedes Register krankt an solchen Problemen. Es wird mit erheblichem Aufwand eine grosse Menge an Daten erhoben, wobei zahlreiche Daten, wie beispielsweise Laborwerte, objektiv sind. Es gibt aber auch Daten, bei denen subjektives Ermessen der Datenmanager einfliesst. Es gibt zwar präzise Anleitungen, wie die Befunde zu werten sind; wie etwa der Schweregrad von Rippenserienfrakturen oder der Knochen- und Gewebeschaden einer offenen Oberschenkelfraktur zu codieren sind. Dennoch bleibt Spielraum für eine gewisse Subjektivität. Trotz allem wurde von einer Zertifizierungsagentur in einem Audit bestätigt, dass die Datenqualität des «Swiss Trauma Registry" gut ist. Zurzeit laufen umfangreiche Vorarbeiten, um eine möglichst identische Quantifizierung der Daten zu erreichen, damit der subjektive Spielraum der Codierenden möglichst minimal wird.

Das «Swiss Trauma Board» ist mit seinem Register auf Kurs und stets bestrebt, dessen Qualität laufend zu optimieren - denn dies ist die Grundlage für Fortschritte in der Behandlung der uns anvertrauten Patientinnen und Patienten.

\section{Bildnachweis sowie Mittelwerte}

Swiss Trauma Board, Jahresbericht 2020, enthält Zahlen des Jahres 2019. Die Jahresberichte sind für die Öffentlichkeit nicht zugänglich.

\section{Literatur}

1 Interkantonale Vereinbarung über hochspezialisierte Medizin. www.gdk-cds.ch/fileadmin/docs/public/gdk/themen/hsm/01 ivhsm_cimhs_14032008_d.pdf

2 Beschluss über die Zuteilung der Leistungsaufträge im Bereich der hochspezialisierten Medizin (HSM): Behandlung von Schwerverletzten. www.gdk-ds.ch/fileadmin/docs/public/gdk/themen/hsm/ HSM-Bereiche/1_2527_d.pdf

3 Fey MF. Wo steht die hochspezialisierte Medizin in der Schweiz? Schweiz Ärzteztg. 2021;102(8):300-3.

4 Moos RM, Sprengel K, Jensen KO, Jentzsch T, Simmen HP, Seifert B, Ciritsis B, Neuhaus V, Volbracht J, Mehra T. Reimbursement of care for severe trauma under SwissDRG. Swiss Med Wkly. 2016 146:w14334.

5 Bless N, Jakob M. Traumazentren in der Schweiz . SWISS KNIFE. 2014;4:10-1.

6 Simmen HP. Interview in SWISS KNIFE. SWISS KNIFE. 2017;1:9.

7 Scheidegger D. Triagekriterien für schwerverletzte Patientinnen und Patienten. SWISS KNIFE. 2017;1:6-8. 